\title{
PRACTICAL APPLICATION OF TURBO EQUALIZATION TO UNDERWATER ACOUSTIC COMMUNICATIONS
}

\author{
Jun Won Choi*, Thomas J. Riedl*, Kyeongyeon Kim*, Andrew C. Singer*, and James C. Preisig** \\ *University of Illinois at Urbana-Champaign \\ **Woods Hole Oceanographic Institute
}

\begin{abstract}
In this paper, we describe linear turbo equalizers (TEQ) and investigate their practical application to underwater acoustic communications. Owing to the ability to achieve a good performancecomplexity trade-off, linear TEQ is a good candidate for long reverberant channels, which usually demand high computational complexity. First, we reveal a relationship between two different TEQ structures; channel estimate (CE)-based minimum mean square error (MMSE) TEQ versus direct-adaptive linear TEQ. We show that without inclusion of the second-order a priori statistics, the coefficients of direct-adaptive TEQ converge to linear timeinvariant form, though an optimal MMSE solution derived from $a$ priori information is time-variant. Nevertheless, the direct-adaptive TEQ yields performance comparable to the CE-based MMSE TEQ while maintaining lower complexity. This was confirmed through real experiments conducted off the coast of Martha's Vinyard, MA ("SPACE 08"). We also discuss a practical design of a multi-channel least mean square (LMS) TEQ and experiments show that the LMSTEQ successfully decodes data achieving up to $19.53 \mathrm{kbit} / \mathrm{s}$ for 1000 meter distance.
\end{abstract}

Index Terms - turbo equalization, underwater acoustic communication, LMS, MMSE, MIMO

\section{INTRODUCTION}

Bandwidth-efficient digital communications over underwater acoustic channels are challenged by channel characteristics of poor quality such as long reverberation due to slow sound propagation and dynamic variation of the channel state in time. To overcome such difficulties, it is crucial to develop effective equalization techniques that can cope with long channel span at a feasible complexity.

Motivated by the "turbo principle", a turbo equalizer (TEQ) exchanges soft information with a channel decoder in an iterative fashion in efforts to approach bit error rate (BER) optimal performance. An initial form of the linear TEQ was the direct-adaptive form [1], where soft symbol estimates are fed back to the equalizer whose coefficients are adjusted such that mean squared error is minimized. The structure of the linear TEQ was investigated in [2], where given the known channel and a priori probabilities on the symbols, an optimal linear equalizer was derived in a minimum mean square error (MMSE) sense. Since channel state is not known in practice, it has to be separately estimated and the estimate is used in the equalizer. In [3], in order to reduce the effects of channel estimation error, second-order statistics of the channel estimation error were incorporated in the equalizer. The application of TEQ to underwater acoustic communication was considered in [4] and an intensive experimental study on TEQ is presented in [5]. Recently, the structure of TEQ was extended to the case of a multiple transmitter system and space-time coding [6].

Most of the above TEQ techniques can be categorized into two structures; 1) channel estimate (CE)-based TEQ and 2) directadaptive TEQ. ${ }^{1}$ Both TEQ approaches show performance loss due to excessive mean square error (MSE) introduced in adaptive channel estimation and equalization operations, respectively. In addition to this, we show that the coefficients of the direct-adaptive TEQ converge to a sub-optimal solution, due to the inability to capture time-varying second-order statistics drawn from a priori information on transmit symbols. The performance of these two types of TEQs are compared in the experimental results. Next, we discuss the practical design of a least mean square (LMS) TEQ (LMS-TEQ) for underwater acoustic communications. We demonstrate through long-term underwater experiments that the LMS-TEQ achieves satisfactory performance while maintaining a reasonable complexity.

\section{SYSTEM DESCRIPTION}

In this section, we describe the structure of transmitter and receiver. The information bits, $\left\{b_{k}\right\}$ are encoded by a rate $1 / 2$ channel encoder, producing the coded bit sequence $\left\{c_{i}\right\}$. The sequence $\left\{c_{i}\right\}$ is permuted using a random interleaver and $Q$ interleaved bits are mapped to a finite alphabet symbol. The interleaved bit sequence is divided into $M$ parallel substreams using a serial to parallel converter. If we let $\widetilde{c}_{m, q}^{t}$ be the $q$ th coded (and interleaved) bit in the $m$ th substream to be transmitted at the symbol time $t$, the successive sets of $Q$ coded bits $\widetilde{c}_{m, 1}^{t}, \cdots, \widetilde{c}_{m, Q}^{t}$ are individually modulated (mapped) to the $2^{Q}$-ary transmitted signal $x_{t}^{m}$. The symbol $x_{t}^{m}$ is sent through the $m$ th transducer. After demodulation and assuming perfect synchronization, the $L \times 1$ received vector $\mathbf{r}_{n}$ is expressed as

$$
\mathbf{r}_{n}=\sum_{k=-K_{f}}^{K_{p}} \mathbf{H}_{n, k} \mathbf{x}_{n-k}+\mathbf{w}_{n},
$$

where $\mathbf{w}_{n}$ is the $L \times 1$ noise vector and $\mathbf{x}_{n}$ is the $M \times 1$ transmit vector at the time index $n$. The $L \times M$ matrix $\mathbf{H}_{n, k}$ is the channel matrix whose $(l, m)$ th element is the complex gain from the $m$ th transmit transducer to the $l$ th receive hydrophone. In this model, we assume that the channel response spans at most $K_{f}+K_{p}+1$ symbols. Due to the interleaver, we assume that the interleaved bits and corresponding symbols are mutually uncorrelated.

\footnotetext{
${ }^{1}$ We do not include joint channel estimation and equalization techniques in this category due to relatively high computational complexity for long channel responses.
} 


\section{TURBO EQUALIZATION WITH LINEAR STRUCTURE: CE-BASED MMSE-TEQ VERSUS DIRECT ADAPTIVE TEQ}

Based on the model (1), the complex-domain relationship between the transmitted symbol and received data can be represented by

$$
\mathbf{y}_{n}=\mathbf{H}_{n} \mathbf{s}_{n}+\mathbf{n}_{n}
$$

where

$$
\begin{aligned}
\mathbf{y}_{n} & =\left[\mathbf{r}_{n+N_{f}}^{T}, \cdots, \mathbf{r}_{n-N_{p}}^{T}\right]^{T}, \mathbf{n}_{n}=\left[\mathbf{w}_{n+N_{f}}^{T}, \cdots, \mathbf{w}_{n-N_{p}}^{T}\right]^{T}, \\
\mathbf{s}_{n} & =\left[\mathbf{x}_{n+K_{f}+N_{f}}^{T}, \cdots, \mathbf{x}_{n-K_{p}-N_{p}}^{T}\right]^{T}, \\
\mathbf{H}_{n} & =\left[\begin{array}{ccccc}
\mathbf{H}_{n+N_{f}, l-K_{f}} & \cdots & \mathbf{H}_{n+N_{p}, l+K_{p}} & \mathbf{0} & \mathbf{0} \\
\mathbf{0} & \ddots & \ldots & \ddots & \mathbf{0} \\
\mathbf{0} & \mathbf{0} & \mathbf{H}_{n-N_{p}, l-K_{f}} & \cdots & \mathbf{H}_{n-N_{p}, l+K_{p}}
\end{array}\right] .
\end{aligned}
$$

For the sake of brevity, we let $\mathbf{s}_{n}=\left[s_{n, 1}, \cdots, s_{n, K}\right]^{T}, \mathbf{y}_{n}=$ $\left[y_{n, 1}, \cdots, y_{n, N}\right]^{T}$, and $\mathbf{H}_{n}=\left[\mathbf{h}_{n, 1}, \cdots, \mathbf{h}_{n, K}\right]$, where $K=$ $M\left(K_{p}+K_{f}+N_{p}+N_{f}+1\right)$ and $N=L\left(N_{p}+N_{f}+1\right)$. The noise vector $\mathbf{n}_{n}$ is assumed to be jointly Gaussian whose covariance matrix is $\mathbf{R}$.

\subsection{CE-based MMSE Turbo Equalizer}

Given $\mathbf{y}_{n}$, a channel matrix $\mathbf{H}_{n}$ and a priori probabilities on $\mathbf{s}_{n}$, a linear MMSE estimate of $s_{n, k}$ for all $k \in\left[M\left(K_{f}+N_{f}\right)+\right.$ $\left.1, M\left(K_{f}+N_{f}\right)+M\right]$ is given by [2]

$$
\hat{s}_{n, k}^{\mathrm{MMSE}}=\mathbf{z}_{n}^{H}\left(\mathbf{y}_{n}-\mathbf{H}_{n} \overline{\mathbf{s}}_{n}+\mathbf{h}_{n, k} \bar{s}_{n, k}\right),
$$

where

$$
\mathbf{z}_{n}=\left(\mathbf{H}_{n} \boldsymbol{\Sigma}_{n} \mathbf{H}_{n}^{H}+\left(1-\sigma_{n, k}^{2}\right) \mathbf{h}_{n, k} \mathbf{h}_{n, k}^{H}+\mathbf{R}\right)^{-1} \mathbf{h}_{n, k},
$$

the vector $\overline{\mathbf{s}}_{n}=\left[\bar{s}_{n, 1}, \cdots, \bar{s}_{n, K}\right]^{T}$ is a priori estimate of $\mathbf{s}_{n}$, and $\boldsymbol{\Sigma}_{n}=\operatorname{diag}\left(\sigma_{n, 1}^{2}, \cdots, \sigma_{n, K}^{2}\right)$ is a covariance matrix of $\mathbf{s}_{n}$ derived from a priori probabilities. To prevent early limit cycle behavior, the LMMSE estimate in (3) does not rely on a priori information on $s_{n, k}$. The CE-based MMSE-TEQ obtains the symbol estimate $\hat{s}_{n, k}^{\mathrm{CE}-\mathrm{MMSE}}$ by replacing $\mathbf{H}_{n}$ by the channel estimate $\widetilde{\mathbf{H}}_{n}$ in (3) and (4). Based on Gaussian approximation of the MMSE estimation error, we can compute an extrinsic LLR for $\left\{\widetilde{c}_{m, q}^{t}\right\}$ as

$$
\begin{aligned}
L_{\text {ext }}\left(\widetilde{c}_{m, q}^{t}\right) & =\ln \frac{P\left(\hat{s}_{n, k}^{\mathrm{CE}-\mathrm{MMSE}} \mid \widetilde{c}_{m, q}^{t}=1\right)}{P\left(\hat{s}_{n, k}^{\mathrm{CE}-\mathrm{MMSE}} \mid \widetilde{c}_{m, q}^{t}=0\right)} \\
= & \ln \frac{\sum_{\theta \in \Theta_{q}^{1}} \exp \left(-\frac{\left|\hat{s}_{n, k}^{\mathrm{CE}-\mathrm{MMSE}}-\mu_{k} \theta\right|^{2}}{\sigma_{r, k}^{2}}\right)}{\sum_{\theta \in \Theta_{q}^{0}} \exp \left(-\frac{\left|\hat{s}_{n, k}^{\mathrm{CE}-\mathrm{MMSE}}-\mu_{k} \theta\right|^{2}}{\sigma_{r, k}^{2}}\right)},
\end{aligned}
$$

where $\Theta_{q}^{1}$ and $\Theta_{q}^{0}$ are the set of all constellation points such that the $q$ th bit is 0 and 1 , respectively, and $\mu_{k}$ and $\sigma_{r, k}^{2}$ are $\mathbf{z}_{n}^{H} \mathbf{h}_{n, k}$ and $\mathbf{z}_{n}^{H} \mathbf{h}_{n, k}\left(1-\mathbf{z}_{n}^{H} \mathbf{h}_{n, k}\right)$ [2]. Since the MMSE TEQ was derived under the assumption that a channel state is known, channel estimation errors degrade the performance of the TEQ. Refer to [2] for the detailed description on the iterative detection and equalization procedure that follow.

\subsection{Direct-Adaptive Turbo Equalizer}

Without a channel estimate, the symbol $s_{k}$ can be directly estimated by direct-adaptive TEQ. By inspecting the optimal MMSE estimate in (3), we can choose the structure of the direct-adaptive TEQ as

$$
\hat{s}_{n, k}^{\mathrm{DA}-\mathrm{TEQ}}=\mathbf{f}^{H} \mathbf{y}_{n}+\mathbf{g}^{H}\left[\begin{array}{c}
\overline{\mathbf{s}}_{n, 1: k-1} \\
\overline{\mathbf{s}}_{n, k+1: K}
\end{array}\right],
$$

where the $M \times 1$ vector $\mathbf{f}$ and the $(K-1) \times 1$ vector $\mathbf{g}$ are the feedforward and feedback coefficients of TEQ, respectively and we let $\overline{\mathbf{s}}_{n, i: j}=\left[\bar{s}_{n, i}, \cdots, \bar{s}_{n, j}\right]$. The soft symbol estimates except that of $s_{n, k}$ are incorporated into the direct-adaptive TEQ through the feedback filter. Note that $M$ separate filters $\{\mathbf{f}, \mathbf{g}\}$ should be applied to obtain $\hat{s}_{n, k}^{\mathrm{DA}-\mathrm{TEQ}}$ for $k \in\left[M\left(K_{f}+N_{f}\right)+1, M\left(K_{f}+N_{f}\right)+M\right]$.

In order to analyze the equalizer, we consider $\bar{s}_{n, j}$ as a random signal, not an expectation. The coefficients $\mathbf{f}$ and $\mathbf{g}$ are adapted such that the mean square error $E\left[\left|s_{n, k}-\hat{s}_{n, k}\right|^{2}\right]$ is minimized. If we consider the LMS algorithm, the weight update equation for $\mathbf{f}$ and $\mathbf{g}$ is given by

$$
\left[\begin{array}{l}
\mathbf{f}^{(n+1)} \\
\mathbf{g}^{(n+1)}
\end{array}\right]=\left[\begin{array}{l}
\mathbf{f}^{(n)} \\
\mathbf{g}^{(n)}
\end{array}\right]+\mu\left(s_{n, k}-\hat{s}_{n, k}\right)\left[\begin{array}{c}
\mathbf{y}_{n} \\
\overline{\mathbf{s}}_{n, 1: k-1} \\
\overline{\mathbf{s}}_{n, k+1: K}
\end{array}\right]
$$

where $\mu$ is a step size and $\mathbf{f}^{(n)}$ and $\mathbf{g}^{(n)}$ are the coefficient vectors after the $n$th update. For a training period, a training symbol can be used in place of $s_{n, k}$ in (8) while for a detection period, tentative decisions can be used for $s_{n, k}$.

Assuming that the channel is locally time-invariant, i.e., $\mathbf{H}_{n}=$ $\mathbf{H}_{m}=\mathbf{H}$ for small $|n-m|$, the coefficients $\mathbf{f}$ and $\mathbf{g}$ approach a steady-state,

$$
\begin{aligned}
\mathbf{f}_{0} & =\left(\mathbf{H}(\mathbf{I}-\mathbf{\Phi}) \mathbf{H}^{H}+E\left[\left|\bar{s}_{n, k}\right|^{2}\right] \mathbf{h}_{k} \mathbf{h}_{k}^{H}+\mathbf{R}\right)^{-1} \mathbf{h}_{k} \\
\mathbf{g}_{0} & =-\left[\begin{array}{ll}
\mathbf{H}_{1: k-1} & \mathbf{H}_{k+1: K}
\end{array}\right]^{H} \mathbf{f}_{0},
\end{aligned}
$$

where $\boldsymbol{\Phi}=\operatorname{diag}\left(E\left[\left|\bar{s}_{n, 1}\right|^{2}\right], \cdots, E\left[\left|\bar{s}_{n, K}\right|^{2}\right]\right)$ and $\mathbf{H}_{i: j}=$ $\left[\mathbf{h}_{i}, \cdots, \mathbf{h}_{j}\right]$. This can be easily derived from the orthogonality principle [7]. From (7), (9), and (10), the direct-adaptive TEQ converges to $\hat{s}_{k}^{\mathrm{DA}-\mathrm{TEQ}, \mathrm{SS}}$ given

$$
\hat{s}_{k}^{\mathrm{DA}-\mathrm{TEQ}, \mathrm{SS}}=\mathbf{f}_{0}^{H}\left(\mathbf{y}_{n}-\mathbf{H} \overline{\mathbf{s}}_{n}+\mathbf{h}_{k} \bar{s}_{n, k}\right) .
$$

Comparing (3), (4) and (11), we see that the structure of the optimal MMSE TEQ and direct-adaptive TEQ are similar but the coefficients $\mathbf{z}$ and $\mathbf{f}_{0}$ are different. Note that (4) is a function of the instantaneous covariance estimate $\boldsymbol{\Sigma}_{n}$ obtained from a priori LLRs while (11) is a function of the covariance matrix $\boldsymbol{\Phi}$ of the signal $\overline{\mathbf{s}}$. Hence, the coefficients of the LMS TEQ approach a locally time-invariant filter $\mathbf{f}_{0}$ while the optimal MMSE filter $\mathbf{z}$ changes in time due to the timevarying matrix $\boldsymbol{\Sigma}_{n}$. Hence, the coefficients of LMS-TEQ do not approach the optimal MMSE solution but to the sub-optimal one which is obtained by replacing $\boldsymbol{\Sigma}_{n}$ by $\mathbf{I}-\boldsymbol{\Phi}$ in (4). However, this gap will get smaller with more iterations as a priori information becomes more reliable, i.e., $\boldsymbol{\Sigma}_{n} \rightarrow \mathbf{0}$ and $\boldsymbol{\Phi} \rightarrow \mathbf{I}$.

\section{LMS TURBO EQUALIZER}

For symbol rates around to a few $\mathrm{k} \mathrm{sym} / \mathrm{s}$ and nominal distance ranges $(50-1500 \mathrm{~m})$, the length of channel responses span up to 100 symbol periods for typical underwater channels. In addition, in order to compensate large signal attenuation, we can combine up to 10 
receive hydrophones. As the problem dimension of the system under consideration in (2) is $K\left(=M\left(K_{p}+K_{f}+N_{p}+N_{f}+1\right)\right)$, the dimension grows rapidly, with nearly one thousand parameters. While the CE-MMSE TEQ is not feasible for such scenarios, the LMSTEQ can be a practical candidate due to linearly growing complexity with problem dimension.

We next show how the LMS-TEQ can be realized for underwater communications. First, to compensate the poor tracking property of LMS algorithm under time-varying underwater channels, we partition the processing block into sub-blocks and repeat the adaptation of the equalizer over the sub-block until the filter weights converge to the steady-state. At each repetition (called 'pass'), the step size, $\mu$ is decreased by the factor of $\rho(<1)$. Note that for each pass, the equalizer coefficients are initialized by the last update in the previous pass. In the absence of a channel estimate, the LMS-TEQ cannot compute $\mu_{k}$ and $\sigma_{r, k}^{2}$ using (5) directly. From (2) and (11), we have

$$
\begin{aligned}
\hat{s}_{n, k}^{\mathrm{DA}-\mathrm{TEQ}, \mathrm{SS}} & =\mathbf{f}_{0}^{H}\left(\mathbf{H}\left(\mathbf{s}_{n}-\overline{\mathbf{s}}_{n}\right)+\mathbf{h}_{k} \bar{s}_{n, k}+\mathbf{n}_{n}\right) \\
& =\mathbf{f}_{0}^{H} \mathbf{h}_{k} s_{n, k}+\eta_{n, k} \\
& =\mu_{k} s_{n, k}+\eta_{n, k},
\end{aligned}
$$

where $\eta_{n, k}$ is the term including the residual interference and noise. From (14), $\mu_{k}$ and $\sigma_{r, k}^{2}$ can be estimated as

$$
\begin{aligned}
\hat{\mu}_{k} & =\sum_{n=1}^{T} \frac{\hat{s}_{n, k}^{\mathrm{DA}-\mathrm{TEQ}}}{Q\left(\hat{s}_{n, k}^{\mathrm{DA}-\mathrm{TEQ}}\right)} \\
\hat{\sigma}_{r, k}^{2} & =\sum_{n=1}^{T}\left|\hat{s}_{n, k}^{\mathrm{DA}-\mathrm{TEQ}}-\hat{\mu}_{k} Q\left(\hat{s}_{n, k}^{\mathrm{DA}-\mathrm{TEQ}}\right)\right|^{2},
\end{aligned}
$$

where $Q(\cdot)$ is the slicer operation that maps the input to the nearest constellation point and $T$ is the length of the processing block.

\section{EXPERIMENTS}

In this section, we evaluate the performance of different linear TEQs via experiments.

\subsection{Experiment Description}

The experiment is conducted off the coast of Martha's Vinyard, MA during Oct. 14th - Nov 2nd, 2008. For data generation, a recursive systematic convolutional (RSC) code with a generator polynomial $(23,35)$ and a random interleaver of the size 19200 38400 are used. For MIMO transmission, two data streams that are spatially multiplexed are transmitted through two source transducers spaced by $50 \mathrm{~cm}$. For the SIMO case, only one transducer is active. The carrier frequency is set to $13 \mathrm{kHz}$ and there is no movement of the transmitter and receiver. Each packet consists of a 400 training symbol period and 1200 data symbol period. The turbo iteration runs over 6 packets. A square-root raised cosine filter with a roll-off factor 0.2 is used both in the transmitter and receiver. A total of 149 data files each of which contains one minute data are transmitted every two hours. Each transmission is indexed with "epoch 1 149". The vertical hydrophone array contains 12 elements spaced apart by 12 $\mathrm{cm}$ and is deployed at ranges of 1000 meters away from the source.

For synchronization and channel length estimation, 1000 symbols of preamble were inserted every 0.2 second. First, rough estimates of sampling time and data sync are computed based on the magnitude of the correlation between the received data samples and the preamble. Then, we estimate the channel length, i.e., $K_{f}+$
$K p+1$ by searching over range of hundreds of symbols around the data sync. This can be done by comparing the magnitudes of the correlation with the threshold, which is adjusted based on relative magnitude levels both in silent and data periods. Noise power is also estimated during silent period. We choose $L_{p}=K_{f}+7$ and $L_{f}=K_{p}+7$ for equalizer length.

\subsection{Results}

In Fig. 1, the channel estimates for the QPSK system is illustrated. A colormap is used to represent the channel gains over the time-delay domain. A recursive least square (RLS) channel estimator is employed over 20000 symbol periods. We observe that the main arrival appears at around the 40th tap and channel gains change dynamically over time. For most of cases, the actual channel length was between 60-100 taps.

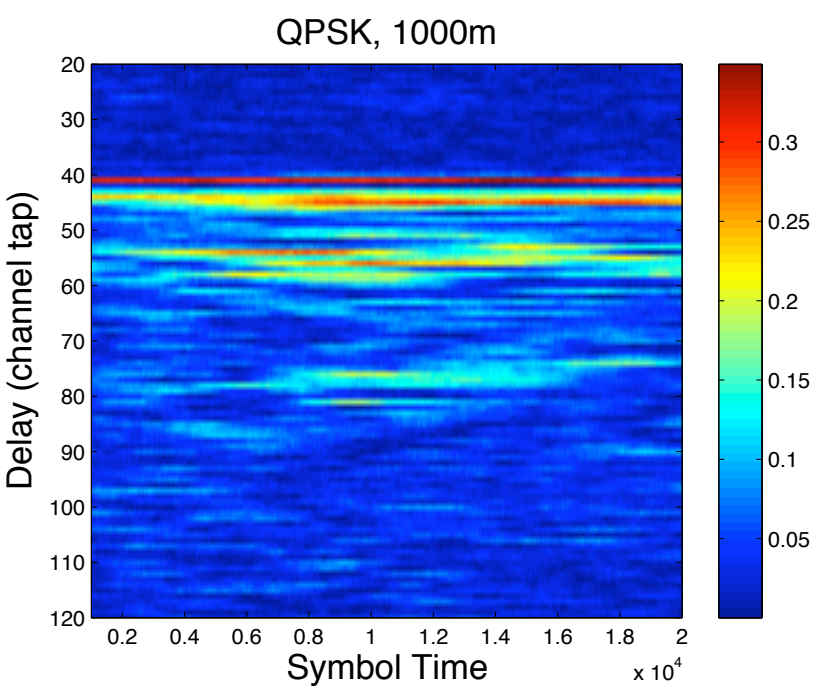

Fig. 1. The plot of channel estimates over 20000 symbol periods.

In Fig. 2, the performance of CE-MMSE-TEQ and LMS-TEQ are compared for 16-QAM SIMO transmission during the epochs $8-18$. The data is transmitted at $9.77 \mathrm{k} \mathrm{sym} / \mathrm{s}$. The 10 receive hydrophones are combined and the performance is measured after seven turbo iterations. For CE-MMSE-TEQ, an LMS channel estimator is employed to estimate a channel during the training period and then the channel is tracked using hard-decision symbol estimate during the remaining period. For LMS-TEQ, a normalized LMS algorithm is used with a step size $\mu=0.1$ which is decreased by the factor of $\rho=0.8$ for every pass. For every sub-block, we run 5 passes in total. As a base-line algorithm, we also include LMS decision-feedback equalizer (LMS-DFE) [8] for comparison. The two TEQs outperform the LMS-DFE for all cases considered. During the epochs 8-12, both CE-MMSE-TEQ and LMS-TEQ retrieve the transmitted data without errors. For the epochs after 8, the BER begins to increase for all equalizers. Both TEQs result in comparable performance for most epochs.

We also take a look at the performance of the LMS-DFE for different symbol rates and modulation orders. The results for epochs 109-114 are provided in Table I. Only the SIMO case is considered. After five iterations, the LMS-DFE can retrieve the data without errors for all BPSK and QPSK cases. This implies that our system can achieve $19.53 \mathrm{k} \mathrm{bit} / \mathrm{s}$ rate (corresponding to the case of QPSK, 


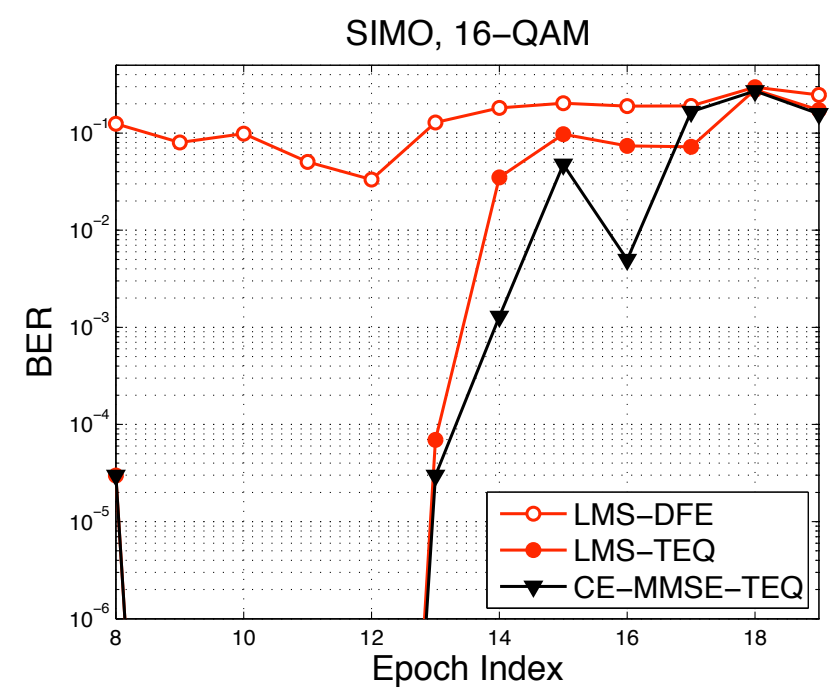

Fig. 2. BER versus epoch index for 16-QAM, SIMO data transmission.

$19.53 \mathrm{k} \mathrm{sym} / \mathrm{s}$ ) with low error probability. On the other hand, for the 16-QAM case, we could achieve perfect data recovery only for $6.51 \mathrm{k} \mathrm{sym} / \mathrm{s}$ case. Note that the QPSK, $19.53 \mathrm{k} \mathrm{sym} / \mathrm{s}$ case and the 16-QAM, $9.77 \mathrm{k} \mathrm{sym} / \mathrm{s}$ case offer the same data rate. Based on this observation, we see that to achieve good spectral efficiency, transmission at higher symbol rates can be more effective than increasing modulation order.

Table 1. The performance of the LMS-TEQ for different symbol rates and modulations.

\begin{tabular}{|c||c|r|r|r|r|}
\hline Mod. & sym/s & No Iter. & Iter. 1 & Iter. 2 & Iter. 5 \\
\hline \multirow{3}{*}{ BPSK } & $6.51 \mathrm{k}$ & 0.0 & 0.0 & 0.0 & 0.0 \\
\cline { 2 - 6 } & $9.77 \mathrm{k}$ & 0.0 & 0.0 & 0.0 & 0.0 \\
\cline { 2 - 6 } & $19.53 \mathrm{k}$ & 0.0002 & 0.0 & 0.0 & 0.0 \\
\hline \hline \multirow{3}{*}{ QPSK } & $6.51 \mathrm{k}$ & 0.0 & 0.0 & 0.0 & 0.0 \\
\cline { 2 - 6 } & $9.77 \mathrm{k}$ & 0.0 & 0.0 & 0.0 & 0.0 \\
\cline { 2 - 6 } & $19.53 \mathrm{k}$ & 0.310 & 0.004 & 0.002 & 0.0 \\
\hline \hline & $6.51 \mathrm{k}$ & 0.01 & 0.0 & 0.0 & 0.0 \\
\cline { 2 - 6 } 16-QAM & $9.77 \mathrm{k}$ & 0.0211 & 0.0138 & 0.0108 & 0.0098 \\
\cline { 2 - 6 } & $19.53 \mathrm{k}$ & 0.48 & 0.49 & 0.51 & 0.55 \\
\hline
\end{tabular}

Next, we show the performance of the LMS-TEQ for a $10 \times 2$ MIMO setup. We do not include that of the CE-MMSE-TEQ due to its high complexity. The BER measured over the epochs 8-31 for several iterations is shown in Fig. 3. In five iterations, the LMS-TEQ could decode the data perfectly except the epochs 18-20. Clearly, over the whole epoch region of interest, the LMS-TEQ outperforms the LMS-DFE.

\section{CONCLUSIONS}

In this paper, we have investigated linear turbo equalizers for underwater acoustic communications. Specifically, we focus on the application of the direct-adaptive LMS-TEQ which has much lower complexity than the channel estimate-based MMSE TEQ. We showed that the direct-adaptive TEQ approaches a sub-optimal solution but

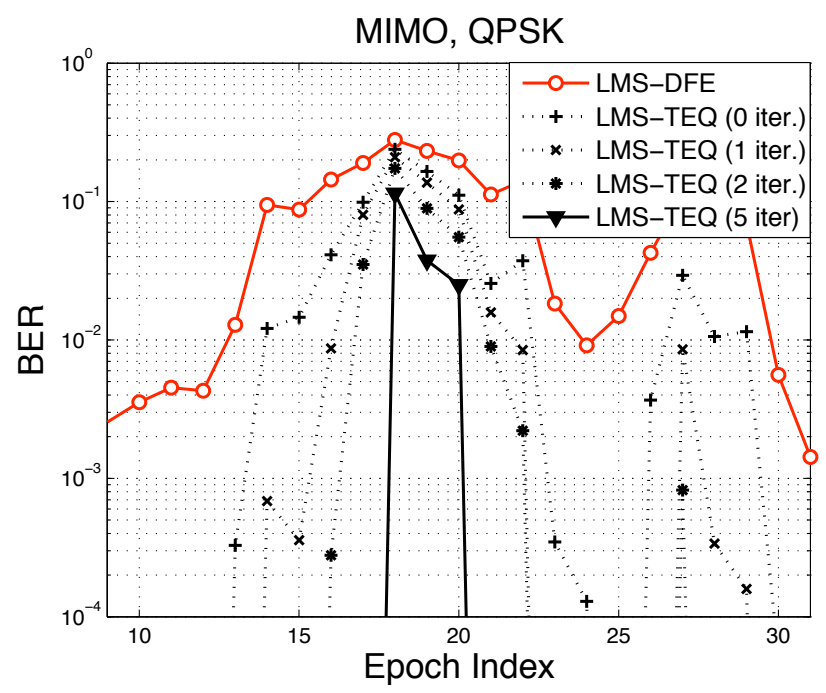

Fig. 3. BER versus epoch index for QPSK, MIMO data transmission.

can be closer to the optimal MMSE solution as more iterations are passed. Based on a comparative study on the CE-MMSE-TEQ and LMS-TEQ, we observe that LMS-TEQ can achieve a performance comparable to that of CE-MMSE-TEQ. In addition, the LMS-TEQ can achieve $19.53 \mathrm{k}$ bit/s without errors over at least 20 minutes of data transmission.

\section{REFERENCES}

[1] C. Laot, A. Glavieux, and J. Labat, "Turbo equalization: adaptive equalization and channel decoding jointly optimized," IEEE Journal on Selected Areas of Commun., vol. 19, pp. 1744-1751, Sep. 2001.

[2] M. T. Tuchler, R. Koetter, and A. C. Singer, "Turbo equalization: principles and new results," IEEE Trans. Commun., vol. 50, pp. 754-767, May 2002.

[3] R. Otnes and M. Tuchler, "Iterative channel estimation for turbo equalization of time-varying frequency-selective channels," IEEE Trans. Wireless Commun., vol. 3, pp. 1918-1923, Nov. 2004.

[4] T. Oberg, B. Nilsson, N. Olofsoon, M. L. Nordenvaad, E. Sangfelt, "Underwater communication link with iterative equalization," IEEE Oceans 2006, pp. 1-6, Sep. 2006.

[5] R. Otnes and T. H. Eggen, "Underwater acoustic communications: long-term test of turbo equalization in shallow water," IEEE Jounral of Oceanic Enginnering, vol. 33, pp. 321-334, July 2008.

[6] S. Roy, T. M. Duman, V. McDounald, and J. G. Proakis, "Highrate communication for underwater acoustic channels using multiple transmitters and space-time coding: receiver structures and expeimental results," IEEE Journal of Oceanic Engineering, vol. 32, pp. 663-688, July 2007.

[7] S. Haykin, "Adaptive filter theory: Third edition" Prentice Hall, 1996.

[8] M. Kocic, D. Brady, and M. Stojanovic, "Sparse equalization for real-time underwater acoustic communications," Proc. OCAEANS, Oct. 1995, pp. 1417-1422. 